\title{
Evaluation of Three Esthetic Restorative Materials Used for Carious or Non- carious Cervical Lesion Restoration
}

\author{
Gabriela Beresescu1, Emanuela Tegla1, Despina Temistocle², Alina Ormenisan¹, Alina Baldean² \\ 1 University of Medicine, Pharmacy, Science and Technology, Târgu Mureş, Romania \\ 2 County Emergency Hospital, Târgu Mureş, Romania
}

\section{CORRESPONDENCE}

Emanuela Tegla

Str. Gheorghe Marinescu nr. 38

540139 Târgu Mureş, Romania

Tel: +40756488817

E-mail: teglaemanuela@yahoo.com

\section{ARTICLE HISTORY}

Received: January 3, 2019

Accepted: January 21, 2019
Gabriela Beresescu • Str. Gheorghe Marinescu nr. 38, 540139 Târgu Mureş, Romania. Tel: +40 265215 551, E-mail: gabriela.beresescu@gmail.com

Despina Temistocle • Str. Gheorghe Marinescu nr. 50 540136 Târgu Mures, Romania. Tel: +40 265212 111, E-mail: despinaluciana@yahoo.com

Alina Ormenisan - Str. Gheorghe Marinescu nr. 38 540139 Târgu Mureş, Romania. Tel: +40 265215 551, E-mail: ormenisanalina@yahoo.fr

Alina Baldean - Str. Gheorghe Marinescu nr. 50, 540136 Târgu Mureș, Romania. Tel: +40 265212 111, E-mail: alina.cocan@gmail.com

\begin{abstract}
Background: Cervical lesions appear on the cervical surface of the lingual or buccal side of the tooth and are classified into carious and non-carious lesions. Aim: The present study evaluates the performance of three different types of aesthetic restorative materials, used for the restoration of carious or non-carious cervical lesions. Materials and methods: The study comprised 195 cervical lesions in 45 patients. The restorations were carried out for non-carious cervical lesions in $34.62 \%$ of the cases, for primary carious lesions in $40.00 \%$ of the cases, and to replace a previous restoration in $25.38 \%$ of the cases. The restorations were evaluated at 2 weeks (the reference line), and then at 1 and 2 years after placement. The following have been assessed: restoration retention, color harmonization, surface texture, margin discoloration, anatomical contour, margin integrity, and the presence of secondary caries. The characteristics were registered in conformity with the modified USPHS criteria. Results: At the one-year evaluation, we noticed the loss of 12 restorations, and after 2 years, the loss of 19 restorations. The results showed significant differences between restorative materials regarding color, margin adaptation, margin coloration, surface texture, as well as criteria regarding the anatomical contour ( $p<0.05)$. Conclusions: The evaluation of the success of restorative material retention must consider the location of the cervical lesion. A successful treatment depends particularly on a full understanding of the factors that caused the lesions and on the method of their treatment.
\end{abstract}

Keywords: carious, non-carious, cervical lesions, restoration materials, retention rates

\section{INTRODUCTION}

Cervical lesions appear on the cervical surface of the lingual or buccal side of the tooth. Conventionally, from an etiological point of view, they are classified into carious and non-carious lesions. ${ }^{1,2}$

Nowadays, non-carious cervical lesions represent a serious problem for oral healthcare. It is well known that they can be caused by abrasion through brushing. In the last twenty years, it has been hypothesized that the etiological factor of these lesions with angular shape could be found in the dental flexion, which appears as a result of tensile stress. Non-carious cervical lesions are classified into 
abrasions, erosions, and abfractions. ${ }^{3,4}$ Grippo et al. used the term abfraction to refer to the pathological loss of tooth substance at the cervical level. ${ }^{5}$ He reported several cases with predisposition to the formation of abfraction-type lesions. Shafer, Hine, and Levy maintain that abrasion and erosion are two distinct, separate processes, each leading to the loss of tooth substance. ${ }^{6}$ A study carried out by Bader et al. showed that the development of cervical lesions has a multifactorial etiology, which includes diet, brushing style, as well as occlusal mechanisms, and that it can have independent effects on various points on the same tooth. ${ }^{7}$ In a longitudinal study that lasted fourteen years, Pintado et al. showed that there is a correlation between occlusal wear and the development of non-carious cervical lesions. ${ }^{8}$ In most cervical lesions, the cavity presents mixed margins, positioned in the enamel and in the dentine, and/or in the cement. As a consequence, the restoration of this type of cavities is more difficult due to the lack of a material which adheres equally to both the enamel and the dentine.

The most challenging problems are posed by the reliability of restorations set with the margin in the dentine. For several years, glass ionomer cements have been thought to be the best materials for the reparation of cervical lesions, because they can form a chemical reaction with both the enamel and the dentine, and can release fluoride for long periods of time, thus having a protective effect against caries. Various clinical researchers have highlighted the possibility to restore cervical caries with the use of conventional glass, with great results. ${ }^{2,9,10}$

Recently, much attention has been paid to the new generation of hybrid, photopolymerizable restoration materials, to resin-modified glass ionomer cements, and to polyacid-modified resin-based composites, all having been increasingly used in the treatment of cervical lesions. In the case of resin-modified ionomer cements, the fundamental reaction of acid-base-polymerization is supplemented by a process of photopolymerization. These materials allow an almost instantaneous hardening, thus trying to solve, at the same time, the problem of sensitivity to humidity. Moreover, the positive properties of glass ionomer cements, such as fluoride release, have been preserved or even improved in the hybrid ionomers. ${ }^{11,12}$

In the oral cavity, the performance of restorative materials can be affected by multiple and clinically interactive variables. Although several earlier studies have evaluated the utility of novel esthetic materials in cervical lesion restorations, there are still certain controversies with regard to the material that can ensure the best results when used clinically. ${ }^{11,13,14}$

As a consequence, we conducted a clinical study for a period of two years, at the end of which we analyzed the per- formance of four different materials, namely: conventional glass ionomer cement, two resin-modified glass ionomer cements, and a polyacid-modified resin-based composite. The present study evaluates the performance of three different types of esthetic restorative materials, used for the restoration of carious or non-carious cervical lesions.

\section{MATERIALS AND METHODS}

\section{Study subjects}

The study included 220 cervical lesions in 53 patients, aged between 25 and 63 years. Inclusion criteria were the absence of the following:

- serious medical condition;

- extended carious lesions;

- severe chronic periodontitis;

- xerostomy;

- apical periodontitis or pulpitis.

Before beginning the conservative treatment, the study subjects were informed about the purpose of the study and the necessity of regular dental checkups. Each patient participated voluntarily and signed a written informed consent.

The patients were also informed about eating habits and received instructions on oral hygiene, particularly through practical demonstrations of correct brushing. Each patient was checked twice a year, using an oral health progress record (OHPR).

According to the OHPR:

$-0 / 1$ indicates good oral hygiene;

-2 indicates slight problems by a narrow margin;

$->2$ indicates certain problems which require additional interventions or scaling.

Patients with poor oral hygiene, strong occlusion and/ or tooth wear were excluded from the study.

There were 45 patients left in the study ( 28 women and 17 men), who underwent a total of 195 restorations, the rest of the patients being excluded for various reasons (tooth extraction, emergence of periodontal complications, the patient's absence from the checkup session).

The restorations were carried out for non-carious cervical lesions in $34.62 \%$ of the cases, for primary carious lesions in $40 \%$ of the cases, and to replace a previous restoration in $25.38 \%$ of the cases. All cavities presented mixed margins, both in the enamel and in the dentine (Table 1). 
TABLE 1. The distribution of restorations based on the clinical situation

\begin{tabular}{lc}
\hline Clinical situation & Number of restorations \\
\hline Cervical non-carious lesions & 67 \\
Primary carious lesions & 78 \\
Preexisting restorations & 50 \\
Total & 195 \\
\hline
\end{tabular}

Each patient presented at least two cervical lesions, which were restored with minimum 2 and maximum 4 different materials. The teeth were divided into 4 groups, based on the restoration material used:

a) Group 1: restorations with conventional glass ionomer cement - G1 $(n=30)$;

b) Group 2: restorations with resin-modified glass ionomer cement - G2 $(n=68)$;

c) Group 3: restorations with resin-modified glass ionomer cement - G3 ( $\mathrm{n}=54)$;

d) Group 4: restorations with polyacid-modified resinbased composites - G4 $(n=43)$.

We applied the restoration materials randomly, on incisors, canines, or premolars, either on the mandibular or maxillary arches.

\section{Preparation and pre-treatment of cavities}

All cavities were prepared in advance, and the restorations were applied by a single dentist. Each patient received at least two restorations.

For the present study, none of the restorations were performed under local anesthesia or using a rubber dam. Humidity control was ensured using cotton rolls and saliva suction pumps. In some situations, a gingival retraction cord was also applied, in order to prevent contamination with saliva or blood.

After the preparation of the operation field and the drying of the cavities, the restoration materials were placed in conformity with the producer's instructions. Restoration materials which had a polymerization indicator were photopolymerized with a high-energy light source $\left(550 \mathrm{~W} / \mathrm{cm}^{2}\right)$.

\section{Evaluation of restorations}

The restorations were evaluated using a blind evaluation technique, all restorations being evaluated during the patient's three visits: at 2 weeks (the reference line), and then
TABLE 2. The distribution of restorations based on the affected tooth and arch

\begin{tabular}{lc}
\hline Distribution of restorations & Number of restorations \\
\hline Upper incisors & 58 \\
Upper canines & 25 \\
Upper premolars & 32 \\
Lower incisors & 25 \\
Lower canines & 29 \\
Lower premolars & 26 \\
Total & 195 \\
\hline
\end{tabular}

at 1 and 2 years after the placement. The following parameters were assessed: restoration retention, color harmonization, surface texture, margin discoloration, anatomical contour, margin integrity, and the presence of secondary caries. In order to determine the stability of the material color, intraoral color photographs were taken right after the placement of the material and at each of the patient's visit for a check-up.

During patient follow-up, the restorations were not modified (for example, refined and polished).

The characteristics were registered in conformity with the modified United States Public Health Service (USPHS) criteria (Table 3). ${ }^{3,6}$ The USPHS evaluation ${ }^{3,6}$ is based on several performance criteria, which are applied to all the parameters concerned:

1. Alpha score (A) - ideal from a clinical point of view - clinical situation with a maximum of clinical performance;

2. Bravo score (B) - acceptable from a clinical point of view - all the parameters are satisfactory, and the restoration is acceptable;

3. Charlie score (C) - unacceptable from a clinical point of view - when one or several characteristics require replacement of the restoration;

4. Delta score (D) - loss of restoration or maximal modification of certain characteristics.

Restoration retention rates were assessed in accordance with the guidelines of the American Dental Association (ADA):

Cumulative failure $\%=[(\mathrm{PF}+\mathrm{NF}) /(\mathrm{PF}+\mathrm{RR})] \times 100 \%$

$\mathrm{PF}$ - number of previous failures, before the present procedure

$\mathrm{NF}$ - number of new failures, at the present procedure $\mathrm{RR}$ - number of restorations at the present procedure 


\section{Statistical analysis}

All results were obtained in the form of Alpha, Bravo, Charlie, and Delta scores, and the statistical analysis of the data was conducted using the SPSS 10.0 for Windows software (SPSS Inc., Chicago, IL, USA). The data were analyzed using the Chi-square test and the Fisher test, with a level of significance of $5 \%$. Because a substantial rate of patients presented more than one restoration, the analysis was performed with a non-independent observation criterion.

\section{RESULTS}

A number of 45 patients were left in the study, therefore we were able to examine 195 restorations. The reasons for not including the rest of the restorations in the evaluation were either a crown replacement, or the patient's decision to stop participating in the study, for personal reasons. During the testing period, we did not notice any loss of vitality or any progression of periodontal pathology in the teeth under investigation.

At the one-year evaluation, of the total number of restorations, we noticed the loss of 12 restorations, and after 2 years, the loss of 19 restorations (Figure 1). The total number of placed and lost restorations according to the anatomical placement of the restoration is listed in Table 3. Retention failure at the level of the mandibular arch was higher than in the maxillary one. The mandibular incisors presented a higher rate of retention failure (Table 3 ).

The retention rate of the restorative materials for a period of two years was $70 \%$ in G1, $100 \%$ in G2, $67 \%$ in G3, and $68 \%$ in G4. During the entire period of our study, four secondary caries emerged. Nevertheless, we discovered significant differences between the restorative materials regarding color, margin adaptation, margin coloration,

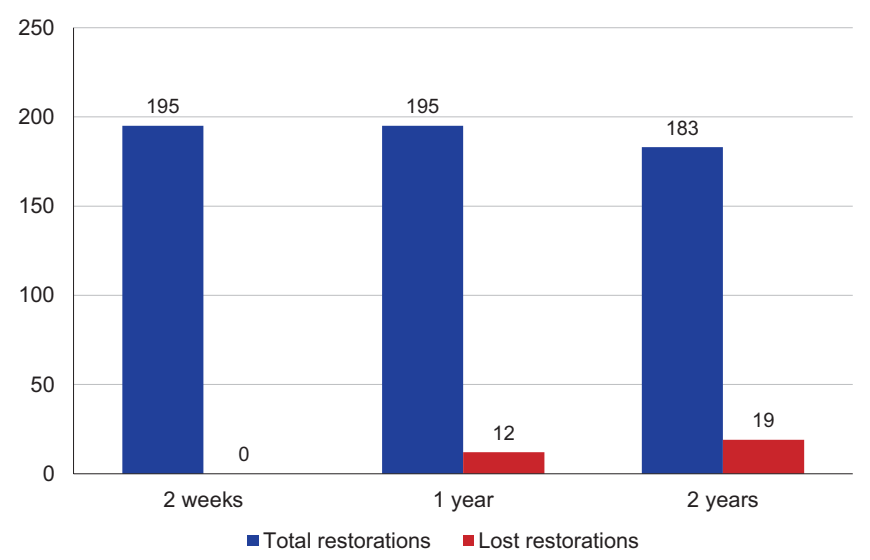

FIGURE 1. The distribution of lost restorations surface texture, as well as the criteria regarding the anatomical contour $(\mathrm{p}<0.05)$ (Table 4$)$.

On the other hand, from the point of view of the retention, G1 presented excellent characteristics compared to the other materials $(\mathrm{p}<0.05)$. Only 2 restorations with conventional glass ionomer cement (G1) received a score of 4 for margin adaptation after 2 years. Despite its higher retention, resin-modified glass ionomer cement (G2) displayed the lowest Alpha scores for margin discoloration, margin adaptation, and anatomical contour, followed by conventional glass ionomer cement (G1) and polyacidmodified resin-based composites (G4) (Table 4).

\section{DISCUSSIONS}

The ADA guide imposes the acceptance of the evaluation of a two-year clinical study and a failure rate of $5 \%$. In the present study, the retention rates of the utilized materials did not exceed this loss percentage. Consequently, we can say that our results demonstrated the efficacy of resinmodified glass ionomer cement (G2) for cervical lesion restorations. Its ionic binding to the structure of the tooth seemed to be more efficient than the binding of other materials tested during our study. ${ }^{15}$

In the present study, we used the modified USPHS criteria for the clinical evaluation of restorations of class $\mathrm{V}$ cavities. Altogether, for the clinical evaluation of tooth restorations, several different protocols have been developed and described, including USPHS, Ryge, or CDA. In order to perform a high-quality evaluation, most evaluation algorithms are based on the classification of quality: retention, anatomical contour, margin integrity, nuance harmonization, and margin discoloration, which can be categorized as acceptable or unacceptable. An objective and reproducible clinical evaluation of tooth restorations can be carried out with the use of any of these classification tools.

TABLE 3. Placed and lost restorations

\begin{tabular}{lccc}
\hline & $\begin{array}{c}\text { Restorations } \\
\text { (n) }\end{array}$ & $\begin{array}{c}\text { Lost } \\
\text { restorations } \\
\text { (n) }\end{array}$ & $\begin{array}{c}\text { Lost } \\
\text { restorations } \\
\text { (\%) }\end{array}$ \\
\hline Upper incisors & 58 & 5 & 5.17 \\
Upper canines & 25 & 3 & 12 \\
Upper premolars & 32 & 1 & 3.12 \\
Lower incisors & 25 & 12 & 48 \\
Lower canines & 29 & 8 & 27.58 \\
Lower premolars & 26 & 3 & 11.53 \\
Total & 195 & 31 & 15.89 \\
\hline
\end{tabular}


TABLE 4. Clinical evaluation of the materials used in the study - USPHS-corresponding scores

\begin{tabular}{|c|c|c|c|c|c|c|c|c|}
\hline & \multicolumn{2}{|c|}{ G1 } & \multicolumn{2}{|c|}{ G2 } & \multicolumn{2}{|c|}{ G3 } & \multicolumn{2}{|c|}{ G4 } \\
\hline & 1 & 2 & 1 & 2 & 1 & 2 & 1 & 2 \\
\hline \multicolumn{9}{|c|}{ Retention } \\
\hline A & 29 & 21 & 68 & 68 & 49 & 36 & 35 & 29 \\
\hline B & 1 & 9 & 0 & 0 & 5 & 18 & 9 & 14 \\
\hline \multicolumn{9}{|c|}{ Color match } \\
\hline A & 30 & 30 & 53 & 24 & 24 & 4 & 39 & 20 \\
\hline B & 0 & 0 & 15 & 44 & 30 & 50 & 4 & 23 \\
\hline \multicolumn{9}{|c|}{ Marginal discoloration } \\
\hline A & 28 & 26 & 45 & 11 & 41 & 27 & 37 & 23 \\
\hline B & 2 & 4 & 23 & 57 & 13 & 27 & 6 & 20 \\
\hline C & 0 & 0 & 0 & 0 & 0 & 0 & 0 & 0 \\
\hline $\mathrm{D}$ & 0 & 0 & 0 & 0 & 0 & 0 & 0 & 0 \\
\hline \multicolumn{9}{|c|}{ Marginal adaptation } \\
\hline A & 30 & 26 & 60 & 27 & 44 & 27 & 35 & 17 \\
\hline B & 0 & 4 & 8 & 26 & 11 & 26 & 9 & 26 \\
\hline C & 0 & 0 & 0 & 0 & 0 & 0 & 0 & 0 \\
\hline \multicolumn{9}{|c|}{ Surface texture } \\
\hline A & 30 & 30 & 49 & 31 & 39 & 24 & 38 & 29 \\
\hline B & 0 & 0 & 19 & 37 & 15 & 30 & 6 & 14 \\
\hline C & 0 & 0 & 0 & 0 & 0 & 0 & 0 & 0 \\
\hline \multicolumn{9}{|c|}{ Anatomic form } \\
\hline$A$ & 29 & 28 & 51 & 29 & 38 & 27 & 30 & 26 \\
\hline $\mathrm{B}$ & 1 & 2 & 17 & 39 & 16 & 27 & 13 & 17 \\
\hline C & 0 & 0 & 0 & 0 & 0 & 0 & 0 & 0 \\
\hline $\mathrm{D}$ & 0 & 0 & 0 & 0 & 0 & 0 & 0 & 0 \\
\hline \multicolumn{9}{|c|}{ Secondary caries } \\
\hline$A$ & 30 & 28 & 68 & 67 & 54 & 54 & 43 & 42 \\
\hline $\mathrm{B}$ & 0 & 2 & 0 & 1 & 0 & 0 & 0 & 1 \\
\hline
\end{tabular}

Restoration loss can be caused by the alteration of dentine binding, by the continuous flexion of the tooth, and by occlusal stress. These factors affect teeth at the level of the maxillary and mandibular arch in a different manner. Researchers have noticed a decrease in retention of cervical lesions due to high flexion in mandibular teeth. ${ }^{16}$

We registered this phenomenon as well, especially in the case of retention in the front mandibular teeth, which reached a level of $47 \%$; despite this, none of the restorations located on the maxillary premolars was lost. Our study also sought to evaluate the success rate of the materials according to different groups of teeth, from the point of view of the occlusion. Therefore, the selection and distribution were random.

Another factor that has been described to affect the retention percentage is the elastic module of materials used for restoration. Heymann et al. showed that the retention frequency in case of restorations with low-level elasticity materials was significantly higher compared to those with a highly elastic module. ${ }^{17}$ In our study, we observed that Group 1 had the highest retention rates, conventional glass ionomer cement having the lowest elastic module of the tested materials. In areas where the occlusal forces are more intensely concentrated, especially at the level of the mandibular incisors, it would be more useful to use restorative materials with a low elastic module, thus increasing the retention rates over time. ${ }^{11,13,18}$

The improvements we noticed regarding the retention of glass ionomer cements were not as significant as we anticipated. The retention rates of various esthetic restorative materials used for the reparation of cervical lesions has been described to be between $69 \%$ and $100 \%$. Retention success depends on the patient's choice, the place and shape of the lesion, but also on the properties of the used materials. Regardless of the material tested, we can expect a high retention rate if the material is set in an area with low concentration of stress. The main reason for the different results of studies might be found in the different 
location of restorations. The differences in retention rates may be due, for example, to the lack of preparation of retentions in the enamel. Moreover, these different results might also be explained by the random distribution of the materials according the groups of teeth and the maxillary or mandibular arch. ${ }^{19,20}$

Regarding the reference line, the high percentage of $\mathrm{Al}$ pha scores (100\%) obtained by resin-modified glass ionomer cement, in the characteristics of the surface may have been due to the protective layer applied over the surface of the material. ${ }^{21}$ This smooth and shiny layer turned into a rough layer by the end of the study. The roughness of the surface may be the result of the very erosive wear of the material, which led to external discoloration, thus tending to have more spots or discoloration than a smooth surface. ${ }^{4,14}$

The anatomical and physiological contour is difficult to reproduce with glass ionomer cements because of their low viscosity and relatively sticky properties. Secondly, the modification in the anatomical contours of the obturation with resin-modified glass ionomer cement might be due to physical-mechanical properties. ${ }^{22}$

According to Levitch et al., occlusal stress, which leads to the distortion of cervical lesions, is the main factor involved in the pathogenesis of cervical lesions. ${ }^{22}$

In our research, strict standards were applied in the evaluation of restorations. An Alpha score was conferred to an excellent restoration. Therefore, most Bravo scores represent the result of this strict rule of observation, while the differences between Alpha and Bravo were only marginal.

Since both Alpha and Bravo show clinical satisfactoriness, all restorative materials that had been used in this study were within adequate limits. Our study is concurrent with the results of previous studies in the fact that the restorative materials used for cervical lesions had different results, in all the aspects of clinical evaluation. According to our results, resin-modified glass ionomer cement (G2), which presented the highest rate of retention, seems to be the most suitable material for the restoration of cervical lesions, although it requires clinical improvements. Nevertheless, the repair of cervical lesions appears to be an ongoing challenge.

\section{CONCLUSIONS}

The evaluation of the success of restorative material retention must consider the location of the cervical lesion. The retention is influenced by several factors, including dental flexion, occlusal stress, dentine surface, and the elastic module of the materials used for restoration. The application of a photopolymerizable cover material can be considered an efficient procedure, especially when the polish- ing procedures are performed right after the restoration. Successful treatment depends particularly on a full understanding of the factors that caused the lesions and on the method of their treatment.

\section{CONFLICT OF INTEREST}

Nothing to declare.

\section{REFERENCES}

1. Gladys S, Van Meerbeek B, Lambrechts P, Vanherle G. Marginal adaptation and retention of glass-ionomer, resin-modified glass ionomers and a polyacid-modified resin composite in cervical class $\mathrm{V}$ lesions. Dent Mater. 1998:14:294-306.

2. Bereşescu G, Bocskay St. Glass ionomer cements used in atraumatic restorative treatment technique. Revista de Medicină şi Farmacie, Universitatea de Medicină şi Farmacie Tg.Mureş. 2009;55:509-510.

3. Ngo H, Mount GJ, Peters MC. A study of glass-ionomer cement and its interface with enamel and dentin using a low-temperature, high-resolution scanning electron microscope technique. Quintessence. 1997;28:63-69.

4. Mount GJ. An atlas of glass-ionomer cements. A clinician's guide. 3rd ed London: Martin Dunitz Ltd, 2002.

5. Grippo JO. Noncarious cervical lesion the decision to ignore or restore. $J$ Esthet Dent. 1992;4:55-64.

6. Neo J, Chew CL, Yap A, Sidhu S. Clinical evaluation of tooth-colored materials in cervical lesions. Am J Dent. 1996:9:15-18.

7. Bader JD, Levitch LC, Shugars DA, Heymann HO, McClure F. How dentists classified and treated non-carious cervical lesions. J Am Dent Assoc. 1993:124:46-54.

8. Pintado MR, Delong R, Ko CC, Sakaguchi RL, Douglas WH. Correlation of noncarious cervical lesion size and occlusal wear in a single adult over a 14-year time span. J Prosthet Dent. 2000;84:436-443.

9. Van Dijken JWV. Durability of three simplified adhesive systems in class $\mathrm{V}$ non-carious cervical dentin lesions. Am J Dent. 2004;17:27-32.

10. Beresescu G, Ormenisan A, Comaneanu RM, Carp Veliscu AM, Manea $\mathrm{MM}$, Ion R. FEM Analysis of stress in non-carious cervical lesion restoration with four different restorative materials. Rev Mat Plast. 2018;55:42-45

11. Yaman SD, Sahin M, Aydin C. Finite element analysis of strength of various resin-based restorative materials in class $V$ cavities. J Oral Rehabil. 2003;30:630-641.

12. Brackett WW, Dib A, Blackett MG, Reyes AA, Estrada BE. Two-year clinical performance of class $\vee$ resin-modified glass-ionomer and resin composite restorations. Oper Dent. 2003:28:477-481.

13. Kubo S, Kawasaki K, Yokota H, Hayashi Y. Five-year clinical evaluation of two adhesive systems in non-carious cervical lesions. J Dent. 2006:34:97-105.

14. Rees JS, Jacobsen $\mathrm{PH}$. The effect of interfacial failure around a class $\checkmark$ composite restoration analysed by the finite element method. J Ora Rehabil. 2000:27:111-116.

15. Xie D, Wu W, Puckett A, Farmer B, Mays J. Novel resin modified glass ionomer cements with improved flexural strength and ease of handling Eur Polym J. 2004;40:343-351

16. Feigal RJ, Musherure P, Gillespie B, Levy-Polack M, Quelhas I, Hebling J. Improved sealant retention with bonding agents: A clinical study of two bottle and single-bottle systems. J Dent Res. 2000;79:1850-1856.

17. Heymann $\mathrm{HO}$, Sturdevant JR, Bayne S, Wilder AD, Sluder TB, Brunson WD. Examining tooth flexure effects on cervical restorations: a two-year clinica study. J Am Dent Assoc. 1991;122:41-47.

18. Pintado MR, Delong R, Ko CC, Sakaguchi RL, Douglas WH. Correlation of non-carious cervical lesion size and occlusal wear in a single adult over a 14-year time span. J Prosthet Dent. 2000;84:436-443.

19. Aranda M, Garcia-Godoy F. Clinical evaluation of the retention and wear of light-cured pit and fissure glass ionomer sealant. J Clin Pediatr Dent. 2015;19:273-277.

20. Croll TP, Nicholson JW. Glass ionomer cements in pediatric dentistry: a review of the literature. Pediatric Dentistry. 2002;24:423-429.

21. Beresescu FG, Hancu V, Mucenis S, Cosarca AS, Comaneanu R, Ormenisan $A$. In vitro study regarding the wearing of glass ionomer cements. Rev Mat Plast. 2015;52:272-274

22. Levitch LC, Bader JD, Shugars DA, Heymann HO. Non-carious cervical lesions. J Dent. 1994:22:195-207. 